\title{
Kurt Cobains Tod: \\ Zur Konstruktion eines paradoxen Generationenhelden
}

\author{
Nicole Falkenbayner
}

\section{Kurt Cobain im Rockbimmel}

Die 2007 von einer sehr bekannten Werbeagentur entworfene Zeitschriftenkampagne für eine Stiefelmarke im Punkstil zeigt in einer Fotomontage den 1994 durch Suizid verstorbenen Grunge-Rocker Kurt Cobain: Als Engel in eine lange weiße Tunika gekleidet, sitzt Cobain auf einer Wolke im Himmel, den Blick verklärt nach oben gewandt, an den Füßen das beworbene Schuhwerk. Der dazugehörige Schriftzug besteht nur aus dem Markennamen mit dem in schmaler Schreibschrift nachgesetzten Wort „forever“. Das Werbebild erhöht nicht nur Kurt Cobain selbst als ,Heiligen' des Punk und als Rocklegende, sondern die Stiefelmarke gleich dazu - denn diese trägt man für immer. „Für immer“ ist aber auch ein Versprechen, sich an den Grunge-Rock zu erinnern, einen Musikstil, der eine ganz bestimmte Jugendkultur symbolisierte und mit der die Figur des toten Kurt Cobain bis heute stark assoziiert wird. Die Reaktion auf die Schuhwerbung - ein Aspekt dieser längst vergangenen Jugendkultur - entsprach jedoch ganz und gar nicht der Erwartung der Werbetreibenden.

Die Werbekampagne wurde nämlich sofort zurückgezogen, nachdem es in den sozialen Medien einen Aufschrei gegen die Stiefelmarke gegeben hatte; sie wurde als geschmacklos bezeichnet. ${ }^{1}$ Diese Anekdote ist ein Beispiel dafür, dass die Emotionen schnell hochkochen, wenn es um die Vermarktung Kurt Cobains geht. Das ist umso erstaunlicher, da die Durchkommerzialisierung ihrer lebenden und toten Ikonen zur Funktionsweise der Populärkultur gehört: Die Iterationen der Bilder von Marylin Monroe, James Dean oder Che Guevara sind seit Jahrzehnten fest in einem global-populären Bildgedächtnis verankert, mit ihnen lassen sich Poster, Tassen und T-Shirts verkaufen. Im Fall von Kurt Cobain evoziert dieser übliche Gebrauch von Bildern aber Irritationen. Die Stellung der Figur Kurt Cobain innerhalb des Kultursystems des Rock war besonders, da die Alternativkultur, der er entstammte, ausdrücklich nicht massentauglich sein wollte. Dass sie es dennoch war, ist einer der Gründe, warum Cobains Tod in einem Band zum Sterben von Helden behandelt wird, denn der frühe Suizid ist Teil der künstlerischen Persona Kurt Cobains, die wiederum durch ihre kurze, aber durchschlagende Präsenz in

1 Mark Sweeney: Saatchi's fired over Kurt Cobain ad, in: The Guardian, Freitag, 5. Mai 2007. https://www.theguardian.com/media/2007/may/25/mcsaatchi.advertising, 25. Mai 2018. 
Medien- und Jugendkultur dazu geführt hat, dass Cobain als Ikone der sogenannten Generation $\mathrm{X}^{2}$ konstruiert, konsumiert und analysiert wurde und weiterhin wird - eine kulturelle Aufladung, zu der in gewissem Maße auch dieser Aufsatz, der nach dem Heroischen fragt, beiträgt.

Cobains Star-Sein entstand im Widerstand gegen das durchkommerzialisierte Mediensystem, das ihn erst zu dem Star machte, der er war. Sein Suizid im Alter von 27 Jahren schrieb ihn endgültig in den Code des Rock'n'Roll ein - den sogenannten „Club of $27 \mathrm{~s}^{\text {“3 }}$ der Protagonisten aus der Rock-Ära des 20. Jahrhunderts, die ihr früher Tod über das normale Star-Sein zu Generationenhelden erhoben hatte: Jim Morrison, Janis Joplin, Jimi Hendrix - Kurt Cobain. Alle folgten in gewisser Weise einem Narrativ der Selbstzerstörung, ${ }^{4}$ über das sie sich gleichzeitig selbst hedonistisch erhöhten und von der meist jugendlichen ,Gefolgschaft' erhöht wurden. Cobains Berühmtheit gründet auf einer Verbindung des Wiedererkennungswertes dieses populärkulturellen Narrativs des Rock-Heros und seiner eigenen Idiosynkrasien. Die Grundstruktur dieses Narrativs lässt sich wie folgt zusammenfassen: Der Held des Rock ist einmalig und einzigartig in seiner Fähigkeit, musikalisch sein Innerstes nach außen zu kehren und sich über permanente Grenzüberschreitungen gesellschaftlicher Normen und Traditionen zu definieren. Musik, Performanz und Selbst fallen idealerweise in eins. Durch Ausreizung aller Möglichkeiten der Bewusstseinserweiterung und Selbstüberschreitung aber folgt auf die Explosion der Kreativität und des Hedonismus die fortschreitende Zerstörung des Selbst, die in Krankheit und frühem Tod, meist in Verbindung mit Drogensucht, endet. Anfang der 1990er Jahre war dieses Narrativ, das in seinen Ausprägungen mit der Counter-Culture der Mitte des 20. Jahrhunderts stark verwoben ist, bereits selbst eine Konvention, die an Attraktionskraft verloren hatte, da sie nicht mehr als ,echt', und damit als direkt affizierend, erlebt werden konnte. Der Rock-Heros war zur gänzlich artifiziellen Figur innerhalb einer postmodernen Verweiskultur geworden. Der Erfolg des Grunge - hier vor allem der Band Nirvana, und damit zuletzt gebündelt in der Figur Kurt Cobains - lag zu einem großen Teil darin, dass Nirvana gerade dieses seit Jahrzehnten eingespielte Narrativ in der Wahrnehmung sowohl der Jugendlichen als auch älterer Kommentatoren über-

2 Diese Generation umfasst die Geburtenkohorten von 1961-1981 innerhalb der westlichen Industriegesellschaften. Der Name leitet sich von dem Episodenroman des Kanadiers Douglas Coupland mit dem gleichen Titel ab, der im Jahr 1991 erschien. Vgl. Tara Barbazan: From Revolution to Revelation. Generation X, Popular Memory and Cultural Studies, London 2017; Douglas Coupland: Generation X. Tales for an Accelerated Culture, Basingstoke 1991; Sherry B. Ortner: Generation X. Anthropology in a Media-Saturated World, in: Cultural Anthropology 13.3, 1998, S. 414-440.

3 Siehe u. a. Eric Segalstad und Josh Hunter: The 27s. The Greatest Myth of Rock \& Roll, Berkeley Lake, 2008.

4 Jesse Kavadlo: The Terms of the Contract. Rock and Roll and the Narrative of SelfDestruction in Don DeLillo, Neal Pollack and Kurt Cobain, in: Studies in Popular Culture 30.1, 2007, S. 87-103. 
schrieb, und zwar paradoxerweise durch einen Anspruch auf Authentizität, der sich durch deheroisierende Gesten ausdrückte.

Im Folgenden soll zunächst begründet werden, warum Diskurse um den Tod Cobains als Heroisierungen gelesen werden können. Nachfolgend wird auf die für die Generation X angenommenen besonderen Gefühlsstrukturen eingegangen und schließlich analysiert, wie Cobain medial und persönlich erinnert wird, und wie er das Narrativ des Künstlersuizids für sich selbst und seine Anhänger gestaltet hat. Cobain, so die These, schuf gerade durch die permanente eigene Deheroisierung seiner selbst die Anlage dafür, ein paradoxer Held einer postheroischen Generation zu werden.

\section{Ist Kurt Cobain ein Held?}

Cobain ist mit den in Freiburg seit 2012 am Sonderforschungsbereich „Helden Heroisierungen - Heroismen" entwickelten Ansätzen als heroische Figur zu beschreiben, ${ }^{5}$ da er - erstens - eine herausragende transgressive Wirkung hatte: Im Sinne des am SFB erforschten ,boundary work ${ }^{66}$ verschob er mit seiner Band Nirvana auf dramatische Art und Weise die ästhetischen und performativen Grenzen dessen, was in den damals vorherrschenden Massenmedien, vor allem im Fernsehen, sichtbar war und werden konnte. Hierfür kann man beispielhaft ein Zitat aus einem Artikel des Musikjournalisten Greil Marcus heranziehen, in der dieser seine eigene Zuschauererfahrung eines Nirvana-Auftritts in einer britischen Unterhaltungssendung beschreibt:

The band completely explodes the context - which means as you watch, nothing fits. More than that, what you are seeing is in some absolute way not right. Every sound, every gesture, is wild, rough and scraping: scraping the paint right off the walls and your knowledge that, in a setting such as this, everything is ritualized, right out of your head. Even as you watch, you can't imagine that this was ever on TV. ${ }^{7}$

In der Geschichtsschreibung des Rock'n'Roll kommt Cobain und seiner Band Nirvana - zweitens - eine besondere Position zu, da der durchschlagende Erfolg des Songs Smells Like Teen Spirit im Jahr 1991 einen grundsätzlichen ,Tonwechsel` in der globalisierten Populärmusik hervorbrachte, die seitdem zumindest in der Außenbetrachtung mit einer sich selbst als abjekt empfindenden Jugendkultur symbolisch zusammengeschlossen wurde: der sogenannten Generation X. In Be-

5 Ralf von den Hoff u. a.: Helden - Heroisierungen - Heroismen. Transformationen und Konjunkturen von der Antike bis zur Moderne, in: helden. heroes. héros. E-Journal zu Kulturen des Heroischen 1.1, 2013, S. 7-14. DOI: 10.6094/helden.heroes/heros./2013/ $01 / 01$.

6 Tobias Schlechtriemen: Der Held als Effekt. Boundary Work in Heroisierungsprozessen, in: Berliner Debatte Initial 29.1, 2018, S. 106-120.

7 Greil Marcus: Artist of the Decade. Kurt Cobain, in: Rolling Stone 812, 1999, S. 46-50, hier S. 49. 
zug auf gerade diese Generation von Helden zu sprechen, mutet zunächst absurd an, denn kaum eine andere Generation wurde so sehr mit einem postmodernen und daher auch postheroischen Zeitgeist identifiziert. ${ }^{8}$ Cobains öffentliche Persona aber entsprach gerade diesem Zeitgeist, der durch einen metareflexiven, zynisch-ironischen Selbsthass gekennzeichnet war, in so außerordentlichem Maße, dass er eine Art der Rezeption auslöste, die den üblichen Status einer Berühmtheit deutlich überschritt und sich vor allem an seinen frühen Tod heftete. Mit Cobain konnten sich entfremdete Jugendliche zumindest im US-amerikanischen, europäischen und ozeanischen Sendebereich von MTV einerseits identifizieren, andererseits trat in seiner Figur dieser Zeitgeist in so extremer Form hervor, dass er auch erschreckend singulär war. Cobain löste ein ,Wir-Gefühl aus, das sich paradox aus der Erkenntnis speiste, dass es kein ,Wir' mehr gab.

Der Tod Cobains war - drittens - ein zentrales, mit semantischen Überschüssen aufgeladenes Medienereignis, das in der sozialen Erinnerung der Generation $\mathrm{X}$ transnational einen festen Platz einnimmt. ${ }^{9}$ Dieser Tod wurde aufgrund der Wirkmacht und Popularität Cobains als Stellvertretertod einer ,durchgeknallten Generation unmittelbar symbolisch gerahmt, wie es etwa Elizabeth Wurzel in ihrer ebenfalls als ,symptomatisch' gelesenen Autobiographie mit dem Titel Prozac Nation beschrieben hat:

On April 8, 1994, as I was completing this book, Kurt Cobain shot himself in the head and was found dead in his Seattle home. His suicide was quickly reduced by much of the media into an example of a more general generational malaise gone completely amok, and references were made to „the bullet that shot through a generation“ $[\ldots]$. Cobain's suicide, despite the extremely private nature of his decision or compulsion to blow his brain out, quickly came to be seen as greatly symbolic. ${ }^{10}$

Während in diesem Zitat auch die Kritik Wurzels an der öffentlichen Nutzbarmachung eines immerhin echten, persönlichen Todes herauszulesen ist, zeigen jüngere Untersuchungen aus den Memory Studies, dass Cobains Tod - und das, wofür er stand - Teil individueller Identitätskonstruktionen innerhalb einer mediatisierten Erinnerungs- und Trauerkultur sind. ${ }^{11}$

Aus dieser Auflistung, die die Figur Kurt Cobains in Bezug zu zentralen Ansätzen der Freiburger Heroisierungsforschung setzt, ergibt sich, dass und wie sich

8 Siehe hierzu Roger Beebe: Mourning Becomes...? Kurt Cobain, Tupac Shakur and the „Waning of Affect“, in: ders. u. a. (Hg.): Rock over the Edge. Transformations in Popular Music, Durham 2002, S. 311-334.

9 Zur Dynamik von Medienereignissen und sozialer sowie kultureller Erinnerung siehe u. a. Astrid Erll / Anne Rigney (Hg.): Mediation, Remediation, and the Dynamics of Cultural Memory, Berlin 2009; in Bezug auf Tod von und Trauer um öffentliche Personen zentral Barbie Zelizer: Covering the Body. The Kennedy Assassination, the Media, and the Shaping of Collective Memory, Chicago 1994.

11 Jennifer Otto Bickerdicke: Fandom, Image and Authenticity. Joy Devotion and the Second Lives of Kurt Cobain and Ian Curtis, Basingstoke 2014; Catherine Strong: Grunge. Music and Memory, London/New York 2011. 
die kulturellen Konstruktionen und Sinnschaffungsprozesse, die den Diskurs um den Tod Kurt Cobains bilden, als Heroisierungsdiskurs lesen lassen. Überraschend ist diese Lesbarkeit von Kurt Cobains Tod als ,Heldentod“ wegen der spezifischen Charakteristika, mit denen seine Generation oft identifiziert wurde: Zynismus, Ironie und weitere Eigenschaften lassen eine grundsätzlich post- oder antiheroische Stimmung vermuten. Überraschend ist diese Lesart aber auch, weil sie darauf hinweisen könnte, dass Heroisierungsprozesse in der Spätmoderne zu einem sehr starken Grad Effekte der Emotionalität sozialer Erinnerung sind auch oder gerade, wenn es eine scheinbar desillusionierte Jugend ist, die sich erinnert. Cobain wäre so die paradoxe Projektionsfläche eines heroisierenden Diskurses als Erinnerungsdiskurs, da er teilweise die Zuschreibungen, für die er dann generationenweit stehen sollte, erst mit hervorbrachte, beziehungsweise einer globalisierten Medienöffentlichkeit vor Augen (und vor allem vor Ohren) stellte, und selbst permanent die eigene Deheroisierung betrieb.

\section{Held der Desillusionierten? Cobain und die Gefüblsstruktur einer mediatisierten Jugendkultur}

Ein Konzept, das von einem der Gründungsväter der britischen Cultural Studies stammt, kann helfen, zu beschreiben, wofür Kurt Cobain als Zentralfigur und heroisiertes Idol stehen könnte. Raymond Williams' Konzept der ,structures of feeling' zielt auf die affektive Dimension der Erfahrung von Lebenswelten, die sich mit nachfolgenden Generationen ändert, und die sich nur unzureichend an festen Formen und Institutionen ablesen lässt:

It is the reduction of the social to fixed forms that remains the basic error. [...] There are the experiences to which the fixed forms do not speak at all, which indeed they do not recognise. [...] Yet the actual alternative to the received and produced fixed forms is not silence: not the absence, the unconscious, which bourgeois culture has mythicized. It is a kind of feeling and thinking which is indeed social and material, but each in an embryonic phase before it can become fully articulate and defined exchange. Its relations with the already articulated and defined are then exceptionally complex. ${ }^{12}$

Wenn man nach der Dynamik fragt, die einen kleinen, von Selbstzweifeln besessenen Mann aus der Provinz des amerikanischen Bundesstaates Washington zum Helden einer Generation werden lassen kann, finden wir eine Art von komplexen Verbindungen zwischen einem vorhandenen, in gewisser Weise festen popkulturellen Narrativ und seiner noch nicht klar geformten Subversion vor. Cobain und Nirvana tauchen innerhalb der Institutionen dieses Narrativs und seiner Kultur auf - in Radio und Fernsehen, in Videoclips, in Interviews und Konzerten - und setzen gleichzeitig scheinbar alles daran, das Skript zu destruieren. In bis dahin noch nicht geformten Irritationen, die durch Cobain und Nir-

12 Raymond Williams: Marxism and Literature, Oxford 1977, S. 128-135. 
vana innerhalb der populärkulturellen Institutionen ausgelöst werden, finden wir Hinweise auf die ,structures of feeling ${ }^{6}$ der sogenannten Generation X, die mit Nirvana und den in ihrem Gefolge zu Popularität gekommenen Musikern an die Oberfläche des Systems der Popmusik geschwemmt werden und dieses kurzzeitig dominieren.

Als Nirvanas Single Smells Like Teen Spirit 1991 den ,King of Pop‘, Michael Jackson, an der Spitze der Billboard Charts ablöst, ändert sich einerseits die Tonalität der Musik selbst von Dur auf Moll, von eher hohen Harmonien zu eher tiefen, unreinen Harmonien. Aber auch der von Cobain halb geschriene, halb gemurmelte Text folgt nicht der gewohnten Hitparaden-Kost der Liebeleien und pauschalen Weltverbesserungsaufrufe, wie sich vor allem am Refrain ablesen lässt:

With the lights out, it's less dangerous

Here we are now, entertain us

I feel stupid and contagious

Here we are now, entertain us. ${ }^{13}$

Vor allem eine Textzeile ist es, die hier besonders den durch Cobain wie durch keinen anderen popularisierten Zeitgeist beschreibt, der in der Folge des Erfolgs von Smells Like Teen Spirit eine ganze Reihe anderer, semantisch ähnlicher Sätze an die Spitze der Charts führt: „I feel stupid and contagious“. Beispielhaft wären hier einige erfolgreiche Künstler zu nennen, deren Popularität weit über die GrungePhase anhielt, deren erste Hitsingles aber eine deutliche Nähe zu Nirvana aufzeigen, und zwar weniger in der musikalischen Ausgestaltung als eben in der ,Gefühlsstruktur', die sich textlich in ihnen ausdrückt: „I'm a creep, I'm a weirdo, what the hell am I doing here, I don't belong here"14, aus dem Refrain des Hits Creep der englischen Band Radiohead, oder des Songs Loser von Beck: „soy un perdedor, I'm a loser, baby, so why don't you kill me“. ${ }^{15}$ Beide Songs aus den Jahren 1992 und 1993 werden in der Hochphase der Popularität von Nirvana veröffentlicht.

An die Stelle fröhlicher Diskoknaben und des positiven Pop-Universalismus, für den die 1980er Jahre stehen, treten entfremdete junge Männer, die sich über ihre Depression, ihr Aufmerksamkeitsdefizit und ihr Verlierertum selbst inszenieren, und dafür von einem jungen Publikum frenetisch gefeiert werden. Dieser zelebratorische Effekt zeigt sich aber eher in den oben zitierten Nachfolgehits anderer Bands als in Cobains Musik selbst: sowohl Creep als auch Loser haben vor allem im Refrain eine nahezu hymnische Qualität, hier wird das Verlierertum also bereits überhöht, was so für Nirvanas Musik nicht zutrifft. Die Widerständigkeit, die in dieser neuen Haltung ausgedrückt wird, entspricht einer Verweigerung permanenter bunter Fröhlichkeit und dem damit verbundenen Erfolgsstreben. Diese Verweigerung wurde aber auch gelesen als Reaktion darauf, dass den

13 Kurt Cobain u. a.: Smells Like Teen Spirit, Single von Nirvana, veröff. 10. September 1991.

14 Thom Yorke u. a.: Creep, Single von Radiohead, veröff. 21. September 1992.

15 Beck Hansen: Loser, Single von Beck, veröff. 8. März 1993. 
Jugendlichen selbst kultureller Sinn und Aufstiegschancen vorenthalten wurden, sie spricht den Verlust einer Kernmotivation der Moderne an: der Hoffnung auf eine bessere Zukunft. ${ }^{16}$

Durch die Popularisierung des Grunge entsteht nun das Paradox, dass eine Alternativszene, die sich vor allem über komplexe Spielarten einer Verweigerungshaltung zu definieren scheint, sich der vollständigen Kommerzialisierung preisgibt. Wie mit vielen anderen musikalischen und populärkulturellen Strömungen wird mit Grunge eine Haltung, die ihre originäre Herkunft in der US-amerikanischen Wandlung zu einer postindustriellen Dienstleistungsgesellschaft hat, medial globalisiert. Das musste zu Reibungen führen, die in der Musik selbst erkennbar sind. Wenn wir nach den ,structures of feeling ${ }^{6}$ der Generation X fragen, scheint hier eben vor allem ein Ton hervor - ein Ton, den ich eingehend mit den Textzitaten beschrieben habe, der sich aber auch im tatsächlichen tonalen System der Musik von Nirvana wiederfindet. Diese Musik enthält Elemente des Punk und Hard Rock, verfügt aber über sehr eingängige Grundstrukturen. Cobains Stil ist gekennzeichnet durch die Gegenüberstellung von sehr laut und sehr leise, Ausbruch und Zurückgenommenheit. Die Texte changieren zwischen Selbstkasteiung, Wut, der Beschreibung unguter Erfahrungen, Ermüdung und Entleerung und setzen gleichzeitig auf Schockelemente.

1991 gilt auch sprichwörtlich als „the year that punk broke“17 - hiermit wird darauf verwiesen, dass die grundsätzliche Haltung dieser kulturellen Sphäre dem Punk entstammt und im kulturellen Code verschiedener Alternativszenen schon lange wirksam ist. Mit dem Erfolg des Grunge, der schnell den Mainstream des populären Musikbusiness erobert und sich vor allem in der Figur Kurt Cobains zentriert, wird ein Narrativ entwickelt, das beschreiben soll, wie eine negative Antihaltung aus dem Untergrund herausbricht und für eine kurze Zeit den Diskurs westlicher kommerzieller Jugendkulturen bestimmt. Bei dieser Dominanz handelt es sich im wahrsten Sinne des Wortes um eine kurze Zeit, denn sie findet ihren Kulminationspunkt bereits wenige Jahre später im Suizid Kurt Cobains am 8. April 1994.

Dass der Suizid Cobains und die Trauer um ihn sich so gut in das Narrativ der Generation X einfügen, liegt an der Spannung zwischen Selbstüberhöhung und Selbstabwertung, die die Grundhaltung der Grunge-Musiker wie auch anderer Künstler der frühen 1990er Jahre ausmacht. Diese Spannung wird zum Kern eines sozialen Erinnerungsnarrativs, das jedoch hauptsächlich medial konstruiert ist - obwohl es sich in persönlich bedeutsamem Verhalten ausdrückte, wie der nächste Abschnitt zeigt.

16 Vergl. z. B. Beebe: Mourning Becomes (Anm. 8), S. 312.

17 Ebd. 


\section{Überböbung oder Normalität: Konstruktionen von Kurt Cobains Suizid als, heroischem' Tod}

In ihrer Monographie Grunge. Music and Memory hat die australische Soziologin Catherine Strong untersucht, wie sich zwanzig Jahre später an die frühen 1990er Jahre und das Erleben dieser Subkultur erinnert wird. Strong fragt danach, wie in der Kommemoration des Grunge unter Menschen mittleren Alters, communities of feeling ${ }^{6}$ entstehen. ${ }^{18}$ Sie stellt dazu Medienanalysen einer Reihe von narrativen Interviews gegenüber, die sie mit australischen Anhängern der Grunge-Kultur führte, die eine, wie Strong es nennt, persönliche Erinnerung an ein „massmediated, global event ${ }^{\text {"19 }}$ hatten. Für die Frage nach heroischen Attributen der Erinnerung an Kurt Cobain ist vor allem Strongs Beobachtung interessant, dass es eine Diskrepanz gibt zwischen der Repräsentation Cobains in medialen Kommemorationszeugnissen und den persönlichen Erinnerungen, die die damals Jugendlichen an ihre persönlichen Reaktionen auf Cobains Tod hatten.

Erstaunlich findet Strong dies vor allem, weil die Erinnerungsnarrative, also die Bedeutungszuschreibungen des Grunge für die Generation X und deren allgemeine Interessen- und Gefühlslage, ansonsten medial und individuell weitgehend deckungsgleich repräsentiert werden und den bereits skizzierten Charakteristika nahezu kanonisch folgen: „respondents' memories of Grunge as a whole are very cohesive, and correspond closely to the way Grunge was and is described in the print media. " ${ }^{20}$ Dies ist nicht mehr unbedingt der Fall, wenn es um persönliche Erinnerungen an Cobains Suizid geht. In ihrer Analyse von Artikeln in Jubiläumsausgaben, die Musikmagazine zum zehnten Todestag Cobains 2004 veröffentlichten, zeigt Strong an mehreren Beispielen, wie in diesem Genre ein postromantisches Überhöhungsnarrativ entwickelt wird, das Cobain entmenschliche:

The anniversary articles on the whole portray Cobain as an exceptional, extraordinary human being. In some cases, he is portrayed as having a status beyond that of human, a supernatural or godlike figure. [...] One article [David Stubbs in Uncut Legends \#2, 2004, S. 25] traces his life, seemingly to work out how he became so „abnormal“, concluding that it „all pointed to a bad end - his family history, his initially high and free spirit suppressed by Ritalin, leaving him a profoundly disaffected, morbidly weird anti-boy“ - that is not one of us. ${ }^{21}$

Cobain, der Künstler, der fundamental anders ist, seltsam und gebrochen Strong analysiert treffend, dass Cobain hier nahezu als Künstlergenie dargestellt wird, eine Darstellungsweise, die sich an romantische Traditionen aus dem 19. Jahrhundert anlehnt und damit das Rock'n'Roll-Skript des tragisch zu früh gestorbenen Musikers übersteigt. Dies ist laut Strong vor allem der Todesart ge-

18

19

20

21

Strong: Grunge (Anm. 11), S. 87.

Ebd., S. 7.

Ebd., S. 85.

Ebd., S. 90-91. 
schuldet, da der Suizid den Tod Cobains mit einer stärkeren kulturellen Wirkmacht ausstattet, als sie den mehr oder weniger unfallhaften Toden durch Überdosierung etwa von Jimi Hendrix, Janis Joplin oder Amy Winehouse anhaftet. ${ }^{22}$ Die mediale Kommemoration hebt also das Übermenschliche, Transgressive an Cobain in besonderer Weise hervor und stilisiert ihn so zu einer Art ,dunklem Künstlerhelden.

Demgegenüber zitiert Strong Aussagen der von ihr Befragten, die vor allem von ihren eigenen Gefühlen nach der Todesnachricht erzählen und abstreiten, Cobain hätte für sie eine Stellung als Held gehabt:

I just remember feeling really shocked, and just feeling a bit empty, not because he was a big hero of mine, I thought he was cute and a good musician, and all that stuff, but, basically, I just thought the circumstances were sad, you know. (Uma)

I just felt this sadness, it wasn't like, oh my god the grunge hero is dead kind of thing, it was just sad. (Lily) ${ }^{23}$

Diese beiden Befragten drücken eine echte, gefühlsmäßige Reaktion auf das Medienereignis der Berichterstattung von Cobains Tod aus: Schock, Traurigkeit und innere Leere werden genannt. Der heroische Status Cobains wird dabei zwar für einen selbst negiert, aber er wird dennoch aufgerufen. Das kann einerseits eine Bezugnahme auf die mediale Darstellung von Cobain als Held der damaligen Jugendlichen darstellen, verweist andererseits aber auch darauf, dass er für Andere (wenn auch nicht für einen selbst) durchaus einen übermenschlichen Status hatte, wie es etwa in einem dritten Kommentar aufscheint, der sogar eine quasi-religiöse Erhöhung nahelegt (der der Interviewte aber selbst - angeblich - nicht anhing):

Nirvana wasn't the religious experience for me, they were just a band I thought was good ... I heard about people who were just, took it as, it's just like the death of Jesus or something. (Trevor) ${ }^{24}$

Paradox lassen sich diese Äußerungen, die Nirvana und Cobain wieder auf den Boden der Realität zurückholen wollen - ,just a band“, „it wasn't like oh my god the grunge hero is dead“ - als Beharren auf der besonderen Qualität lesen, die Cobain möglicherweise so stark verkörpern wollte: Authentizität. Die hier zitierten Nirvana-Fans sind vielleicht als besonders treue Nirvana-Fans zu bezeichnen, als eine Verehrergemeinschaft, die den Verstorbenen so erinnert, wie es seiner eigenen Erinnerungsanweisung entsprach, hatte Cobain doch selbst zu Lebzeiten jegliche Form der Überhöhung scheinbar immer weit von sich gewiesen. Gerade in der Abweisung des Heldenstatus, aber gekoppelt mit dessen permanentem Aufruf, erscheint Cobain so als affektiv besonders aufgeladene Figur.

In weiteren Aussagen, die Strong zitiert, zeigt sich, dass es in der Erinnerung gerade die Ablehnung des Ruhmes ist, Cobains angebliche Normalität und Nähe

22 Ebd., S. 92.

23 Zitiert ebd., S. 95.

24 Zitiert ebd., S. 96. 
in einem kulturellen und kommerziellen Mediensystem, die für die Befragten seine Besonderheit ausmachen. Strongs Befragte beharren implizit auf einem kulturellen Code, der, obwohl er zu einer stark medialisierten Generation gehört, keiner anderen Qualität so viel kulturelles Kapital einräumt wie der Authentizität:

Cobain is being granted authenticity because of both his perceived normality and his ability to express emotions or write about experiences that respondents could relate to. This adds extra weight to his ordinariness, in that respondents feel that if they could relate to what he was expressing, then he could not be too different to them. ${ }^{25}$

Während also die Presse in der Kommemoration die Erhöhung und Heroisierung Cobains über seine fundamentale Andersartigkeit konstruiert, funktioniert der Diskurs des Besonderen innerhalb der Fangemeinschaft - zumindest in der Gruppe, die von Strong interviewt wurde - gerade über die Normalisierung Cobains, die ihn mit einem hohen Maß an Authentizität als dem kulturellem Kapital für das Wertesystem der Generation X ausstattet. Cobain sei eben kein Heiliger, kein Rock-Held, scheint hier hervor, sondern möglicherweise ein echter Held: weil er seinen Fans echt erschien und seine Musik die eigenen Gefühle spiegelte. Hier zeigt sich ein hohes, für die Postmoderne üblicherweise negiertes Maß an Identifikation. Cobain hat dieses kulturelle Kapital selbst ambivalent mitkonstruiert.

\section{Der (Anti-)Held der Selbstzerstörung: Cobains Auslegung des Rock'n'Roll-Narrativs}

Die structures of feeling,, die durch Kurt Cobain als Galionsfigur der Generation $\mathrm{X}$ ausgedrückt werden, sind wegen der sie bestimmenden Ambivalenz, die sich ebenfalls in der Heroisierung von Cobain findet, schwer zu fassen: Es ist eine Ambivalenz zwischen Widerstand, Traurigkeit und vor allem Authentizität einerseits, und Komplizenschaft mit dem globalen Musiksystem und dem Kommerz andererseits. Diese Ambivalenz findet sich in Cobains Darstellungsstrategien zu seinen Lebzeiten durchgängig wieder. Seiner eigenen Überhöhung begegnet er ironisch, ja zynisch. Obwohl er Fans auf Konzerten beschimpft und verhöhnt, die Sprachrohrfunktion zurückweist, bedient er letztere durch sein Verhalten doch ganz ausgezeichnet: durch sein ostentatives, immer wieder betontes Leiden an Bauchschmerzen, Heroinsucht, Depression und durch seine Interviews, in denen er immer wieder auf die Systemfehler patriarchalischer Verhältnisse hinweist, die Homophobie und den Chauvinismus der USA beklagt. So sehr er sich zu mühen scheint, das Gegenteil zu erreichen - Cobain sagt immer, was Medien und Publikum offenkundig hören wollen: Cobain trifft den Zeitgeist.

Vor allem verdanken Nirvana ihren herausragenden Erfolg dem Umstand, dass sie Zugang zur damals größten globalisierten Kommerzmaschine der Populär- 
kultur hatten: MTV. Ein Erfolg wie der von Nirvana wäre auch deswegen heute nicht mehr möglich, weil es nicht mehr diesen einen massenmedialen Kanal gibt, der die Macht hätte, der Jugend weltweit nicht nur einen Musikstil, sondern ein ganzes Lifestylepaket vorzusetzen und vor allem zu verkaufen. Es ist sicher falsch, anzunehmen, Cobain wäre so naiv gewesen, dass er das nicht ganz genau gewusst hätte. Immer wieder scheint er sich auf Arten und Weisen inszenieren zu wollen, auf die er seine Botschaften ,gegen das System ${ }^{6}$ - und gegen das konventionell gewordene Rock'n'Roll-Skript als Teil dieses Systems - einbringen kann.

In den ,structures of feeling', in denen Cobain eine Schlüsselstellung für die Generation X einzunehmen scheint, wird sein Freitod im April 1994 zum finalen Ausweis der immer bedrohten Authentizität und Transgression, für die die Figur des Musikers ja gerade heroisiert wird, und der totalen Konventionalisierung innerhalb eines Medien- und Warensystems, zu dem das Skript des Genrehelden gehört. Dieses Skript evoziert den romantischen Künstler nicht nur über den Tod, wie oben beschrieben, sondern auch dadurch, jedes Gefühl in transgressiver Weise auszudrücken.

Der Abschiedsbrief Cobains evoziert genau diese Dopplung von Authentizitätsanspruch und Übernahme von Konventionen, wie sie für die 1990er Jahre wohl typisch war: Einerseits berühren noch heute seine letzten Worte, in denen er das Glück der kleinen Tochter nur durch das eigene Ableben für überhaupt möglich hält, sowie die Widmung des Briefes an Boddah, den imaginären Freund aus seiner eigenen Kindheit.

Zentral erscheinen das Gefühl einer Korruption, der nostalgische Glaube an die Unschuld der Kindheit und die Furcht vor der Zukunft, die vor allem in der Passage heraussticht, die an Frau und Tochter gerichtet scheint, die zurückbleiben werden:

I have a goddess of a wife who sweats ambition and empathy and a daughter who reminds me too much of what I used to be, full of love and joy, kissing every person she meets because everyone is good and will do her no harm. And that terrifies me to the point to where I can barely function. I can't stand the thought of Frances becoming the miserable, self-destructive, death rocker that I've become. ${ }^{26}$

Andererseits erscheint die Selbstbeschreibung als „miserable death rocker“ ganz dem Narrativ der Selbstzerstörung durch negative Selbstüberhöhung zu entsprechen. Ebenso ambivalent erscheint die kurze Textpassage, die an die Fans gerichtet ist:

Thank you all from the pit of my burning, nauseous stomach for your letters and concern during the past years. I'm too much of an erratic, moody baby! I don't have the passion anymore, and so remember, it's better to burn out than to fade away. ${ }^{27}$

26 https://kurtcobainssuicidenote.com/kurt_cobains_suicide_note.html, 25. Mai 2018.

27 Ebd. 
Im vorliegenden Sammelband werden andere Fälle besprochen, in denen historische Figuren versucht haben, das spätere Gedenken durch eigene letzte Schriften zu präfigurieren. Im Wissen um den baldigen Tod hat etwa ,Scott of the Antarctic ${ }^{\prime}$ in seinen letzten Tagbucheinträgen betont, wie vorbildlich er und seine Männer sich verhalten haben und dass sie als, Gentlemen' zu sterben willens seien. ${ }^{28}$ Natürlich scheint auch in Cobains Brief der Gedanke auf, ein Vermächtnis oder eine Erinnerungsanweisung zu hinterlassen. Aber, in diesem Sinne ganz ähnlich den oben zitierten Songzeilen, herrscht in seinem Brief klar die Geste der Selbsterniedrigung, ja des Selbsthasses vor. Dass Cobain den Lebenswillen verliert, weil er ein erratisches Baby mit Stimmungsschwankungen sei, kann man wohl nur als Geste der Anti-Heroisierung des Selbst lesen. Cobain gibt hier eine Erinnerungsanweisung, die auf Deheroisierung beharrt, sich aber gleichzeitig der eigenen Herausgehobenheit bewusst ist.

Vieles, was popkulturell aus dieser Zeit der 1990er Jahre stammt, und wofür eben gerade Cobain und Nirvana stehen, scheint von einer Konvergenz sich widerstrebender Vorstellungen von Widerständigkeit und Authentizität, Kommerz und Zustimmung charakterisiert.

\section{Held des Postheroischen}

In diesem Aufsatz wurden der Tod Kurt Cobains und die Kommemoration dieses Todes in der Perspektive der aktuellen Heroisierungsforschung gelesen. In Bezug auf die grundsätzlich als postmodern und postheroisch aufgefasste Generation $\mathrm{X}$ und auf Cobains ebenso zu charakterisierenden Habitus scheint dies zunächst kontraintuitiv. Diese Lesart trotzdem vorzuschlagen, kann einen Beitrag zur Frage leisten, auf welche Art und Weise sich Grundmuster des Heroischen und des heroisierten Todes - immer wieder auch und gerade in eine postheroische ,Gefühlslandschaft' einschleichen und unterhalb der Schwelle einer postulierten affektlosen, entkernten kulturellen Sphäre auf paradoxe Art und Weise weiter wirksam sind. ${ }^{29}$ Hierzu wurde auf die mit der Generation X identifizierte Gefühlsstruktur unter Zuhilfenahme des Konzepts der structures of feeling' von Raymond Williams eingegangen. Nach dieser Beschreibung zeichnete der Aufsatz dann für zwei Bereiche nach, wie ambivalente Heroisierungsdiskurse im Fall Cobain operieren: zunächst innerhalb von medial dargestellten und mit einem Generationengefühl zusammengeschlossenen Repräsentationen von Cobain und dessen Tod, für die in Anlehnung an die Arbeit von Strong gezeigt werden konnte, dass das kanonische Mediennarrativ, das Cobain nicht nur in den Code des Rock'n'Roll-Helden, sondern sogar in ein romantisches Künstlernarrativ einschreibt, den Selbstberichten von Fans entgegensteht. Letztere bleiben auch

28 Siehe den Beitrag von Barbara Korte in diesem Band.

29 Siehe hierzu auch die Beiträge von Joachim Grage und Ulrich Bröckling in diesem Band. 
zwanzig Jahre nach seinem Tod dem kulturellen Code des Grunge treu, indem sie die Besonderheit Cobains einerseits bestreiten, über die Investition eigener Gefühle der Trauer, an die sie sich erinnern, jedoch wiederum paradox bestätigen. Der besondere Stellenwert von Authentizität als Haltung des Grunge, die vor allem von Nirvana verkörpert wurde, wird weiterhin betont, auch wenn die damaligen Fans schon längst das mittlere Alter erreicht haben. Diese Form der eigenen Deheroisierung betreibt Cobain in seinem Abschiedsbrief, dem zweiten Bereich, der hier genauer vorgestellt wurde.

So wie Cobain selbst den Spielregeln des Ruhms nie entgehen konnte und immer wieder von ihnen eingefangen wurde, so können offensichtlich auch die Fans, die Cobain den Heldenstatus absprechen und für sich selbst reklamieren, ihn nicht als Held zu verehren, nicht dem Widerspruch entgehen, dass selbst in ihrer postheroischen Haltung der Negation heroische Muster immer wieder zum Tragen kommen. 
